\title{
Albuterol Delivery via Facial and Tracheostomy Route in a Model of a Spontaneously Breathing Child
}

\author{
Brandy Cooper and Ariel Berlinski MD
}

\begin{abstract}
BACKGROUND: Some pediatric patients receiving therapeutic aerosols undergo tracheostomy, and others who are tracheostomized continue requiring inhaled therapies upon decannulation. It is unknown whether a dose adjustment is required. Different devices are available for facial and tracheostomy delivery, and in some instances, the assisted technique is used. We hypothesized that the change from face mask to tracheostomy would result in a decrease in the lung dose. METHODS: A breathing simulator connected in series to a filter holder and an anatomically correct head model of a child was used. The drug captured in the filter was termed the lung dose. Breathing patterns with tidal volumes of 50,155 , and $300 \mathrm{~mL}$ were tested. Albuterol hydrofluoroalkane (pressurized metered-dose inhaler [pMDI]) with an AeroChamber Mini (face and 4.5-mm tracheostomy), AeroTrach (4.5-mm tracheostomy), and AeroChamber (face) and albuterol $(2.5 \mathrm{mg} / 3 \mathrm{~mL})$ with a continuous output nebulizer (face and $4.5-\mathrm{mm}$ tracheostomy) were tested. Masks were used for facial delivery. Four units of each device were tested. Particle size of the pMDI was measured by cascade impaction. Albuterol concentration was determined via spectrophotometry $(276 \mathrm{~nm})$. RESULTS: Switching from facial to tracheostomy delivery increased lung dose with nebulizer (all breathing patterns). When a pMDI was used, lung dose was unchanged or increased for the 50- and $155-\mathrm{mL}$ and decreased for the 300-mL breathing pattern. The use of the assisted technique increased lung dose only during nebulization with the $300-\mathrm{mL}$ breathing pattern. The particle size of the pMDI decreased by 19-23\% when traveling through the tracheostomy tube, which retained $<4 \%$ and $<26 \%$ of the nominal dose of a nebulizer and pMDI, respectively. CONCLUSIONS: The effect of changing the delivery route from face to tracheostomy was variable and depended on the delivery device and the breathing pattern. There is no advantage of using the assisted technique to enhance aerosol delivery. Key words: tracheostomy; drug delivery; aerosol; jet nebulizer; valved-holding chamber; metered dose inhaler; artificial airway. [Respir Care 2015;60(12):1749-1758. (C) 2015 Daedalus Enterprises]
\end{abstract}

\section{Introduction}

Advances in the care of critically ill pediatric patients have led to an increase in the number of patients who

\footnotetext{
Ms Cooper is affiliated with the College of Pharmacy, University of Arkansas for Medical Sciences, Little Rock, Arkansas. Dr Berlinski is affiliated with the Pulmonology Section, Department of Pediatrics, University of Arkansas for Medical Sciences, Little Rock, Arkansas and the Pediatric Aerosol Research Laboratory, Arkansas Children's Hospital Research Institute, Little Rock, Arkansas.
}

Ms Cooper presented a version of this paper at the 48th Annual Fall Seminar September 5, 2014, Arkansas Association of Health-System Pharmacists in Little Rock, Arkansas.

Dr Berlinski has disclosed relationships with Vertex, AbbVie, Aptalis Pharma, Genetech, Janssen Research and Development, Gilead, Teva, Philips, Novartis, and Therapeutic Development Network. The Pediatric Aerosol Research Laboratory at Arkansas Children's Hospital Research Institute was partially established by and receives partial support from the George Endowment for Asthma. Ms Cooper was supported by a Uni- undergo tracheostomy as part of the process of liberation from mechanical ventilation. ${ }^{1,2}$ Many of these patients are treated long-term with inhaled aerosols that are given through the tracheostomy. Although many of these patients could be decannulated in the future, their need for inhaled therapy remains present. Conversely, many patients with chronic pulmonary conditions that receive inhaled therapies might undergo tracheostomy as part of their treatment for worsening in their clinical status. ${ }^{1}$ There are no current guidelines to help practitioners decide whether a dose adjustment is needed when changing the

\footnotetext{
versity of Arkansas for Medical Sciences College of Pharmacy Summer Research Fellowship.

Correspondence: Ariel Berlinski MD, Pediatric Pulmonology Section, 1 Children's Way, Slot 512-17, Little Rock, AR 72202. E-mail: BerlinskiAriel@uams.edu.
}

DOI: $10.4187 /$ respcare. 04142 
administration route of the aerosol from facial to through the tracheostomy and vice versa. ${ }^{2} \mathrm{~A}$ study in adult subjects found no difference in amikacin delivery between both routes. ${ }^{3}$ Moreover, there is no information on whether the delivery of aerosols through the facial route will be affected by the presence of a tracheostomy tube.

Most of our knowledge on optimization of aerosol delivery through tracheostomy derives from in vitro studies. ${ }^{4-9}$ Multiple factors have been reported to affect drug delivery through tracheostomies, such as: type of aerosol generator, type of interface, inner diameter (ID) of the tracheostomy tube, use of bias flow, use of different breathing patterns, and use of the assisted technique. ${ }^{4-9}$ The latter involves the use of a resuscitation bag to provide support breaths while the aerosol is being delivered. A survey about practices on aerosol delivery to spontaneously breathing tracheostomized children showed that many centers used devices/delivery techniques that have been shown to decrease aerosol delivery efficiency through tracheostomies. ${ }^{10}$

Previous studies done in pediatric models of spontaneously breathing children reported that a pressurized metered-dose inhaler (pMDI) with a spacer/valved holding chamber (VHC) and a continuous output jet nebulizer used with the assisted technique delivered the highest amount of albuterol.6,7 The same studies reported that use of the assisted technique was beneficial when used with jet nebulization but detrimental when pMDIs/VHCs were used. Other studies have shown a benefit of using the assisted technique or have been neutral.,5 The particle size of nebulized aerosols is significantly reduced when traveling through pediatric size tracheostomy tubes, but data on aerosols generated by a pMDI/VHC are lacking. ${ }^{7}$

A better understanding of aerosol delivery through tracheostomies is clinically relevant and long-needed. We hypothesize that changing the delivery route from the face to the tracheostomy, using a smaller tidal volume, using a tracheostomy tube with smaller ID, the presence of tracheostomy during drug delivery through the face, and the use of the assisted technique with a pMDI/VHC will result in a lower lung dose. We also hypothesize that use of the assisted technique with a jet nebulizer will result in a higher lung dose and that the particle size of an aerosol generated by a pMDI/VHC will decrease when traveling through a pediatric tracheostomy tube.

\section{Methods}

The experiments were performed at the Pediatric Aerosol Research Laboratory at Arkansas Children's Research Institute, Little Rock, Arkansas.

\section{Breathing Model}

An anatomically correct, 3-dimensionally printed oronasal head model of a 5-y-old child was used. ${ }^{11}$ The head

\section{QUICK LOOK}

\section{Current knowledge}

Aerosol delivery is complicated in spontaneously breathing pediatric patients due to breathing pattern, interface (mask or mouthpiece), and patient cooperation. Chronically ill children with tracheostomies often receive aerosol therapy, yet knowledge regarding delivery or need for dose adjustment is incomplete. The optimal appliance for delivery is also not known.

\section{What this paper contributes to our knowledge}

In a pediatric model, the effect of changing the delivery route from face to tracheostomy was variable and depended on the delivery device and the breathing pattern. There was no advantage in using an assisted technique to enhance aerosol delivery. The particle size of a metered-dose inhaler decreased after traveling through a pediatric tracheostomy tube. A reduction in the internal diameter of a tracheostomy tube impaired aerosol delivery.

model was connected in series to a breathing simulator (PARI Compass, Munich, Germany) with a low dead space filter holder placed between them (Fig. 1A). ${ }^{6}$ The simulator allowed the programing of tidal volume, inspiratory time, and respiratory rate. The following breathing patterns were used: infant (tidal volume, $50 \mathrm{~mL}$; breathing frequency, 30/min; inspiratory-expiratory ratio, 1:3), child (tidal volume, $155 \mathrm{~mL}$; breathing frequency, 25/min; inspiratory-expiratory ratio, 1:2), and an older child (tidal volume, $300 \mathrm{~mL}$; breathing frequency, 25/min; inspiratory-expiratory ratio, 1:2). Once all studies through the face were completed, a hole was drilled in the model to allow the tracheostomy tube insertion. A $4.5-\mathrm{mm} / 6.3-\mathrm{mm}$ ID/outer diameter (Tracoe, Boston Medical Products, Westborough, Massachusetts) was used in all scenarios (Fig. 1B). In addition, a 3.5-mm ID/5-mm outer diameter tube was also used with the infant breathing pattern.

\section{Devices}

A medium AeroChamber ComfortSeal mask (Monaghan Medical, Plattsburgh, New York) was used for facial delivery of a pMDI/VHC (Fig. 2). A Bubbles the Fish II pediatric aerosol mask (PARI Respiratory Equipment, Midlothian, Virginia) was used for facial delivery of nebulized aerosols (Fig. 2).

During facial delivery, 4 units of the AeroChamber Plus and AeroChamber Mini (both from Monaghan Medical) and a continuous output jet nebulizer (Hudson RCI UpDraft II Opti-Neb nebulizer, Teleflex Medical, Research 


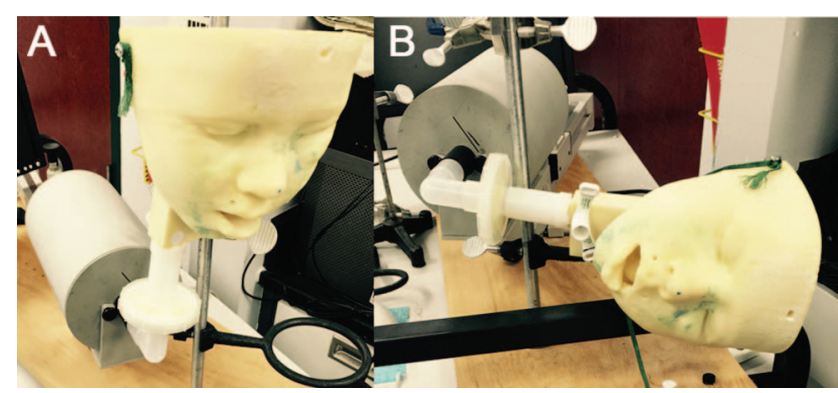

Fig. 1. A: Setup used during facial pMDI delivery. B: Setup used during tracheostomy nebulizer delivery.

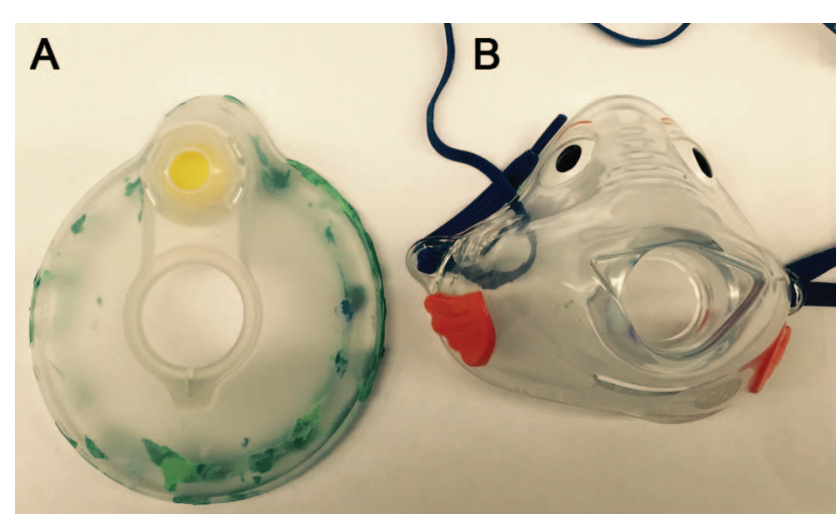

Fig. 2. Masks used during facial aerosol delivery. A: Medium AeroChamber ComfortSeal Mask (Monaghan Medical, Plattsburgh, NY). B: Bubbles the Fish II pediatric aerosol mask (PARI Respiratory Equipment, Midlothian, VA).

Triangle Park, North Carolina) were used (Fig. 3A). The nebulizer was connected to a T-piece that served as interface with the tracheostomy and connected to a $15-\mathrm{cm}$ corrugated tube. The nebulizer and the AeroChamber Mini were operated with both unassisted and assisted techniques (Fig. 3B). ${ }^{10}$ The latter involves the use of a resuscitation bag to provide support breaths while the aerosol is being delivered.

During tracheostomy delivery, 4 units of AeroTrach Plus (Monaghan Medical), AeroChamber Mini, and nebulizer were used (Fig. 3A). The AeroChamber Mini and the nebulizer were both operated using assisted and unassisted techniques (Fig. 3B).6,7

All of the VHCs were made out of antistatic materials. The AeroChamber Plus is a $149-\mathrm{mL}$ holding chamber with a built-in one-way valve. This device is designed to attach to the AeroChamber ComfortSeal mask. The AeroTrach Plus is a $149-\mathrm{mL}$ holding chamber with a built-in one-way valve that is designed to be attached directly to a tracheostomy tube. Both devices allow the use of the pMDI without removing the canister from the plastic actuator. The AeroChamber Mini is smaller $(110 \mathrm{~mL})$ and has an inspiratory one-way valve and an exhalation channel. This device requires that the pMDI be separated from the manufacturer's plastic actuator and placed into the

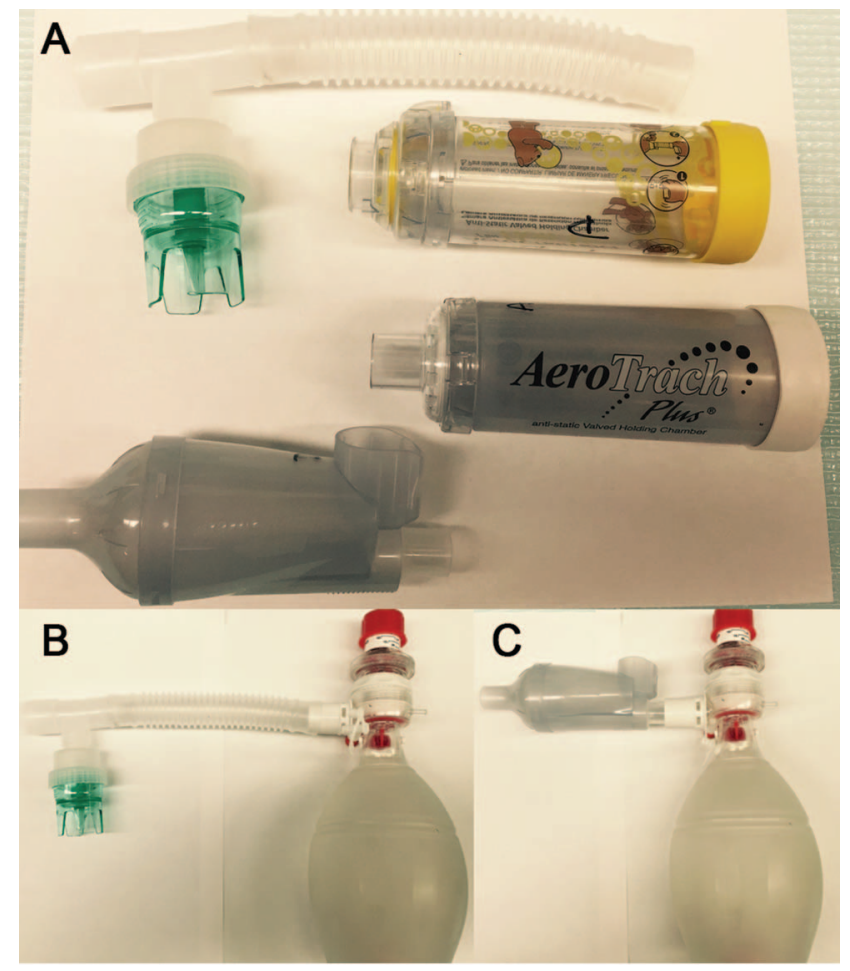

Fig. 3. Devices used during facial and tracheostomy aerosol delivery. A: Devices (from top to bottom): Hudson nebulizer (facial and tracheostomy), AeroChamber (facial), AeroTrach (tracheostomy), and AeroChamber Mini (facial and tracheostomy). B: Configuration used during the assisted technique with a Hudson nebulizer. C: Configuration used with the assisted technique with the AeroChamber Mini.

canister port. This device is marketed for use with the tracheostomy.

A self-inflating resuscitation bag (Pediatric AMBU Spur II, AMBU, Glen Burnie, Maryland) with a stroke volume of $450 \mathrm{~mL}$ was used during the assisted technique (Fig. 3B). During pMDI delivery, 6 consecutive breaths were supported at the beginning of inhalation. During nebulizer delivery, every other breath was supported at the beginning of inhalation for the duration of the nebulization time.

\section{Protocol}

Facial Delivery. A new disposable filter (PARI Respiratory Equipment) was placed in the filter holder before each run. The breathing pattern was then selected in the breathing simulator, and the accuracy of the tidal volume was verified using a mass flow meter (TSI, Shoreview, Minnesota). ${ }^{12}$ The model was used in a vertical position. ${ }^{6}$ The face mask was sealed to the head/airway model with putty, and the VHC was then connected to it. At the beginning of every testing day, the albuterol pMDI (Ventolin hydrofluoroalkane, $90 \mu \mathrm{g}$; GlaxoSmithKline, Brentford, United Kingdom) was primed with 4 puffs. The same 


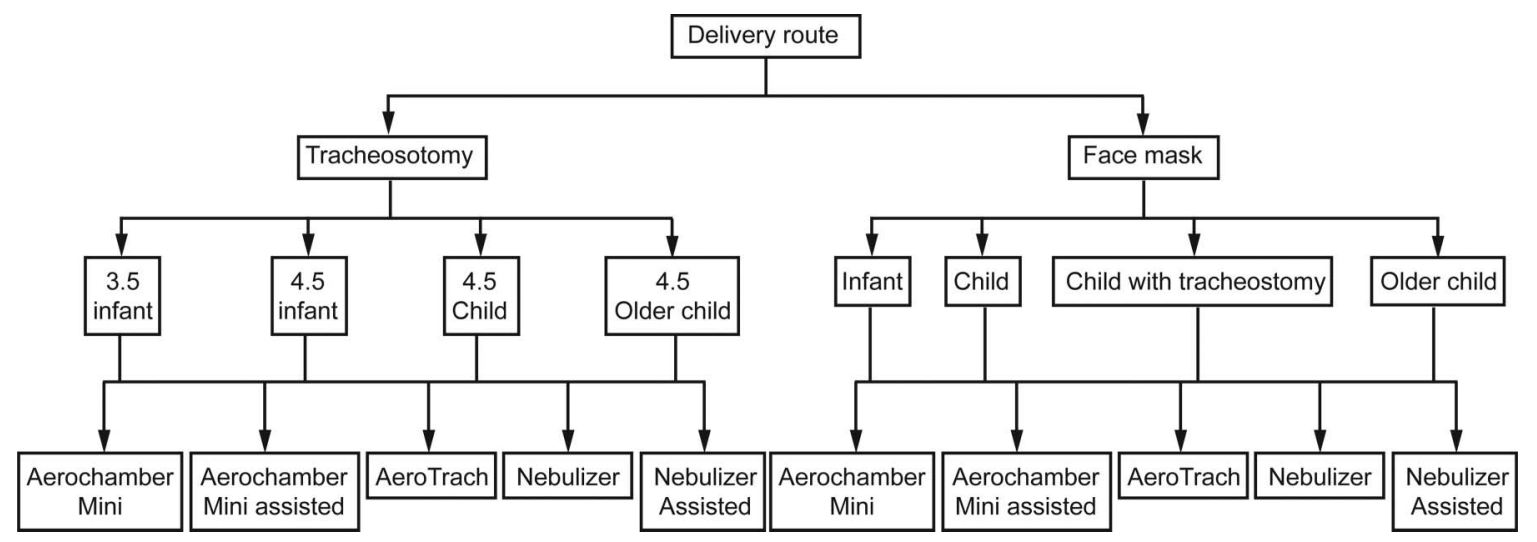

Fig. 4. Experimental map.

canister was used for each run in the series of 4 for the VHC. The canister was not used if it reached zero or if there were not enough puffs to complete the experiment (run of 4). The pMDI was shaken for $5 \mathrm{~s}$ and inserted into the back of the AeroChamber. To improve coordination of actuation, the breathing simulator was set to deliver 7 breaths, and the actuation took place at the beginning of the second inhalation. This process was repeated 9 more times for a total of 10 puffs with the goal of improving drug recovery. The aerosol canister was weighed before and after every 10 puffs as a quality control measure. The filter was then removed from the holder and processed for albuterol concentration. ${ }^{12}$ This process was done for each of the 4 VHCs and was repeated for each of the 3 breathing patterns. The AeroChamber Mini followed the procedure above with the exception of a few modifications. The AeroChamber Mini was attached to the mask using an adapter. The canister was separated from the actuator and placed within the actuator device at the rear top of the AeroChamber Mini. The AeroChamber Mini was operated alone and with the assisted technique as described above. The VHCs were cleaned and air-dried until no residual albuterol was found.

During nebulization, the face mask was sealed and attached to the face model using putty. The face model was rotated laterally to prevent any residual drip from contaminating the filter sample (Fig. 1B). The nebulizer was connected to the mask via its T-piece, and a 6-inch corrugated tube was placed distal to the mask. A filter was placed at the end of the tube to decrease environmental contamination. The computer breathing simulator was then set to run for $5 \mathrm{~min}$, and the nebulizer was operated at a $6-\mathrm{L} / \mathrm{min}$ flow rate for $5 \mathrm{~min}$ for all of the breathing parameters. The nebulizer cup was weighed before each run (dry, after loading the cup with albuterol sulfate nebulizer solution [2.5 mg/3 mL]), at the end of the nebulization period, and after adding $5 \mathrm{~mL}$ of double-deionized water. The amount left in the nebulizer cup was calculated by subtracting the dry weight from the weight after the $5 \mathrm{~mL}$ were added and then multiplying this value by the albuterol concentration. ${ }^{7}$ This method was used as a quality control measure, and since there were no differences, results were not reported. This was done to ensure that each nebulizer cup was operating correctly and that each run received approximately the same amount of drug. This procedure was repeated with 4 different units for the 3 different breathing patterns and operated during spontaneous breathing and with the assisted technique as described above.

Tracheostomy Delivery. When the tracheostomy was used, the procedure was the same as above with a few modifications. Instead of the AeroChamber, we used the AeroTrach holding chamber, which is specifically designed to be adapted to a tracheostomy tube. ${ }^{6}$ The AeroChamber Mini procedure was the same, but the adapter was not needed because the AeroChamber Mini is designed to attach directly to a tracheostomy tube. The nebulizer runs had no change in devices or techniques. ${ }^{6}$ The AeroChamber Mini and the nebulizer were studied during spontaneous breathing and with the assisted technique as described above, whereas the AeroTrach was studied during spontaneous breathing alone. ${ }^{6,7}$ Four different units of each device were tested with all 3 different breathing patterns.

Additional Testing. The delivery of aerosol through the face using the child pattern over a 4.5-mm ID tracheal tube was also tested with the tracheostomy in place. The delivery of aerosol trough a 3.5-mm ID tracheostomy tube was also studied using an infant breathing pattern. A full map of the testing plan can be seen in Figure 4.

\section{Albuterol Determination}

The concentration of albuterol in the filters was determined using the following process. ${ }^{6,7}$ Filters and filter holders were washed with double-deionized water. The filter was placed in a 50-mL tube and was vigorously vortexed 
and shaken. The sample was then analyzed with spectrophotometry at $276 \mathrm{~nm}$ (Biomate $3 \mathrm{UV}$-visible spectrophotometer, Thermo Fisher Scientific, Waltham, Massachusetts). When delivery occurred through the tracheostomy tube, it was flushed with $10 \mathrm{~mL}$ of double-deionized water, and the washings were analyzed for content of albuterol.

\section{Particle Size Determination}

Three different units of albuterol pMDI and 3 different units of Mini and Aero VHCs were tested. A Next Generation Impactor (MSP, Shoreview, Minnesota) assembled with internal and external filter and operated at $30 \mathrm{~L} / \mathrm{min}$ was used to determine particle size of the aerosol leaving the holding chambers alone, the trachea of the face model, and the tracheostomy tube.7,13 A standard United States Pharmacopeia throat was used when measuring the aerosol exiting the VHCs. ${ }^{11}$ The United States Pharmacopeia throat was replaced by the face model that was connected to the Next Generation Impactor via a custom-made adapter (MSP) when measuring the aerosol exiting the trachea. ${ }^{11}$ The same adapter was used with a previously published tracheal model when measuring the aerosol exiting the tracheostomy tube. ${ }^{7}$ Ten actuations of the albuterol hydrofluoroalkane were used with each scenario. All stages of the Next Generation Impactor were washed with doubledeionized water and tested for albuterol concentration. Mass median aerodynamic diameter (MMAD), geometric SD, and percentage of drug mass associated with particles $<5 \mu \mathrm{m}$ were calculated using CITDAS 3.1 software (Copley Scientific, Nottingham, United Kingdom) using the drug recovered from stage 2 to the external filter. ${ }^{7}$

\section{Statistical Analysis}

The amount of drug recovered in the filter was the main outcome and was termed the lung dose. The comparisons of lung doses between delivery through the face and tracheostomy, between the different size tracheostomies used, between facial delivery with tracheostomy in place and no tracheostomy placement, and the between assisted and spontaneous breathing were evaluated with a $t$ test. Analysis of variance followed by Tukey test (multiple comparisons) was used to compare breathing patterns for each device/delivery route scenario. The comparison of particle size generated by the pMDI/VHC alone and through the face and tracheostomy was done by analysis of variance followed by the Dunnett test. $P$ values $<.05$ were considered statistically significant. A statistical software package was used for all of the calculations (Kaleidagraph 4.1, Synergy Software, Reading, Pennsylvania).

\section{Results}

Results are displayed in Tables 1, 2, and 3 and Figure 5.

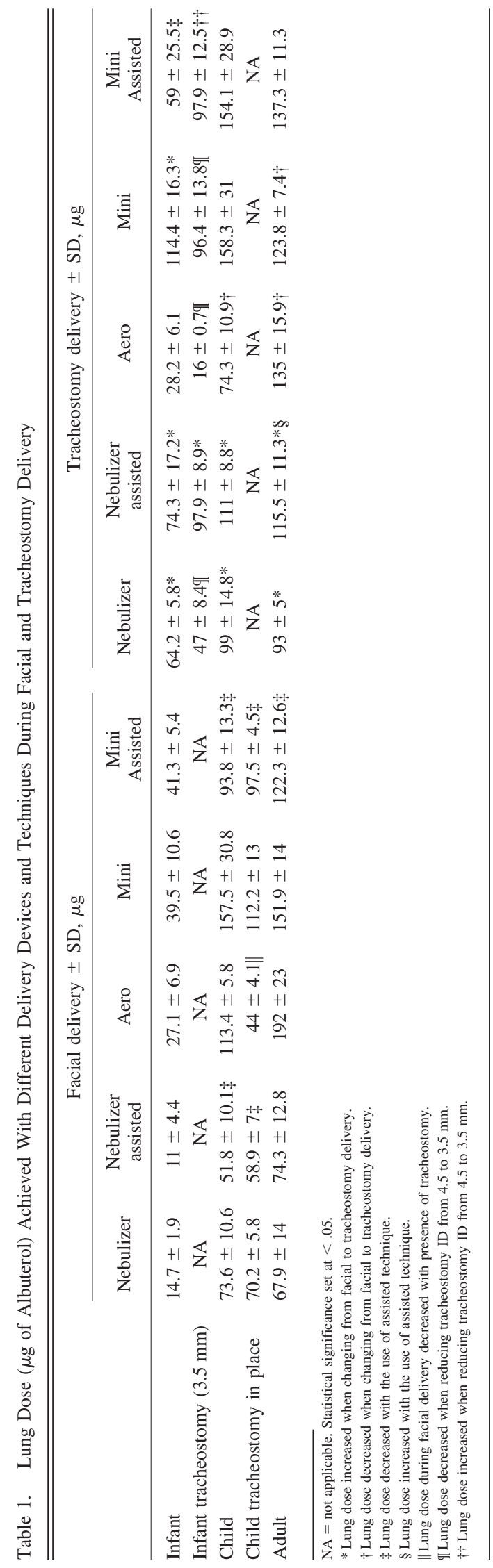


Table 2. Variation in Lung Dose Between Facial and Tracheostomy Delivery Route for Different Devices and Delivery Techniques

\begin{tabular}{|c|c|c|c|c|c|}
\hline & \multicolumn{5}{|c|}{ Tracheostomy/Facial Lung Dose Ratio } \\
\hline & Nebulizer & $\begin{array}{c}\text { Nebulizer } \\
\text { Assisted }\end{array}$ & Aero & Mini & $\begin{array}{c}\text { Mini } \\
\text { Assisted }\end{array}$ \\
\hline Infant & $4.4 *$ & $6.8 *$ & 1 & $2.9 \div$ & 1.4 \\
\hline Child & $1.3 *$ & $2.1 *$ & $0.6 \dagger$ & 1 & $1.6 \div$ \\
\hline Adult & $1.4 *$ & $1.6 *$ & $0.7 \dagger$ & $0.8 \div$ & 1.1 \\
\hline \multicolumn{6}{|c|}{$\begin{array}{l}\text { Boldface numbers represent tracheostomy/facial lung dose ratios that have statistically } \\
\text { significant differences between delivery routes. } \\
* P<.03 \text {. } \\
\dagger P<.004 \text {. } \\
\ddagger P \leq .02 \text {. }\end{array}$} \\
\hline
\end{tabular}

\section{Comparison Between Facial and Tracheostomy Delivery}

Changing the delivery route of nebulized albuterol from facial to through tracheostomy increased lung dose several-fold irrespective of the use of the assisted technique $(P<.03)$. The effect was more pronounced for the infant breathing pattern (tidal volume $50 \mathrm{~mL})(P<.004)$ (Tables 1 and 2 and Fig. 5).

Changing the delivery route of albuterol administered with a pMDI and AeroChamber VHC from facial to through tracheostomy without the assisted technique decreased the lung dose in the child (tidal volume $155 \mathrm{~mL}$ ) and older child breathing pattern (tidal volume $300 \mathrm{~mL})(P=.004$ and $P=.008$, respectively) and did not change it in the infant breathing pattern $(P=.81)$. Changing the delivery route of albuterol administered with the pMDI and AeroChamber Mini VHC without the assisted technique from facial to through the tracheostomy decreased the lung dose in the older child breathing pattern $(P=.02)$, increased it in the infant breathing pattern $(P<.001)$, and did not change it in the child breathing pattern $(P=.97)$.

Changing the delivery route of albuterol administered with the pMDI and AeroChamber Mini VHC with the assisted technique from facial to through the tracheostomy increased the lung dose in the child breathing pattern $(P=.02)$, but it remained unchanged for the infant and the older child breathing patterns $(P=.26$ and $P=.13$, respectively).

\section{Breathing Patterns}

During nebulization through the face without the assisted technique, the infant breathing pattern (tidal volume $50 \mathrm{~mL}$ ) resulted in a lower lung dose than the child (tidal volume $155 \mathrm{~mL}$ ) and older child (tidal volume $300 \mathrm{~mL}$ ) breathing patterns $(P=<.001$ and $P<.001$, respectively) (Table 1 and Fig. 5). When the assisted technique was used, the lung dose obtained with each breathing pattern increased with age $(P<.001)$. During nebulization through the tracheostomy without the assisted technique, the infant breathing pattern resulted in a lower lung dose than the child and older child breathing patterns $(P=<.002$ and $P=.006$, respectively). When the assisted technique was used, the lung dose obtained was similar among all breathing patterns $(P=.07)$.

Using a pMDI with an AeroChamber Mini VHC through the face without the assisted technique, the infant breathing pattern resulted in a lower lung dose than the child and older child breathing patterns, which were similar $(P=<.001)$. When the assisted technique was used, the lung dose obtained with each breathing pattern increased with age $(P<.001)$. When using the pMDI with an AeroChamber VHC through the face without the assisted technique, the lung dose increased with age $(P<.001)$.

When using a pMDI with an AeroChamber Mini VHC through the tracheostomy without the assisted technique, the lung dose was higher for the child than for the infant breathing pattern $(P=.036)$. When using a pMDI with an AeroChamber Mini VHC through the tracheostomy with assisted delivery, the lung dose was lower for the infant breathing pattern than for the child and older child breathing patterns $(P=.0007$ and $P=.003$, respectively). When using a pMDI with an AeroTrach VHC through the tracheostomy without assisted delivery, lung dose increased with age $(P<.0001)$.

\section{Effect of Tracheostomy Size}

During nebulization, the change in tracheostomy size from 4.5 to $3.5 \mathrm{~mm}$ decreased the lung dose in the infant breathing pattern (tidal volume $50 \mathrm{~mL}$ ) without the assisted technique $(P=.02)$ but remained unchanged when the assisted technique was used $(P=.06)$ (Table 1).

During albuterol delivery with a pMDI and either the AeroTrach VHC or the AeroChamber Mini VHC, the change in tracheostomy size from 4.5 to $3.5 \mathrm{~mm}$ decreased the lung dose in the infant breathing pattern without the assisted technique $(P=.004$ and $P<.001$, respectively) but had the opposite effect when the assisted technique was used $(P=.001)$.

\section{Comparison Between Facial Delivery With and Without Tracheostomy in Place}

Delivering nebulized albuterol through the face using the breathing pattern of a child (tidal volume $155 \mathrm{~mL}$ ) did not change the lung dose with the presence of the tracheostomy $(P=.60$ and $P=.30$ for the non-assisted and assisted technique, respectively) (Table 1).

Delivering albuterol via a pMDI/VHC through the face using the breathing pattern of a child did not change the lung dose with the presence of the tracheostomy for the AeroChamber Mini $(P=.62$ and $P=.52$ for the nonassisted and assisted technique, respectively) but decreased for the AeroChamber $(P=.02)$. 
Table 3. Equivalence Between Nebulizer $(2.5 \mathrm{mg} / 3 \mathrm{~mL})$ and Pressurized Metered-Dose Inhaler (Number of Actuations) With Valved Holding Chamber Delivery During Facial and Tracheostomy Delivery

\begin{tabular}{|c|c|c|c|c|}
\hline & \multicolumn{2}{|c|}{ Facial Delivery } & \multicolumn{2}{|c|}{ Tracheostomy Delivery } \\
\hline & AeroChamber & AeroChamber Mini & AeroTrach & AeroChamber Mini \\
\hline Infant & 5.4 & 3.7 & 22.8 & 5.6 \\
\hline Infant tracheostomy $(3.5 \mathrm{~mm})$ & NA & NA & 25 & 4.9 \\
\hline Child & 5.5 & 4.7 & 13.3 & 6.3 \\
\hline Adult & 3.5 & 4.5 & 6.9 & 7.5 \\
\hline
\end{tabular}

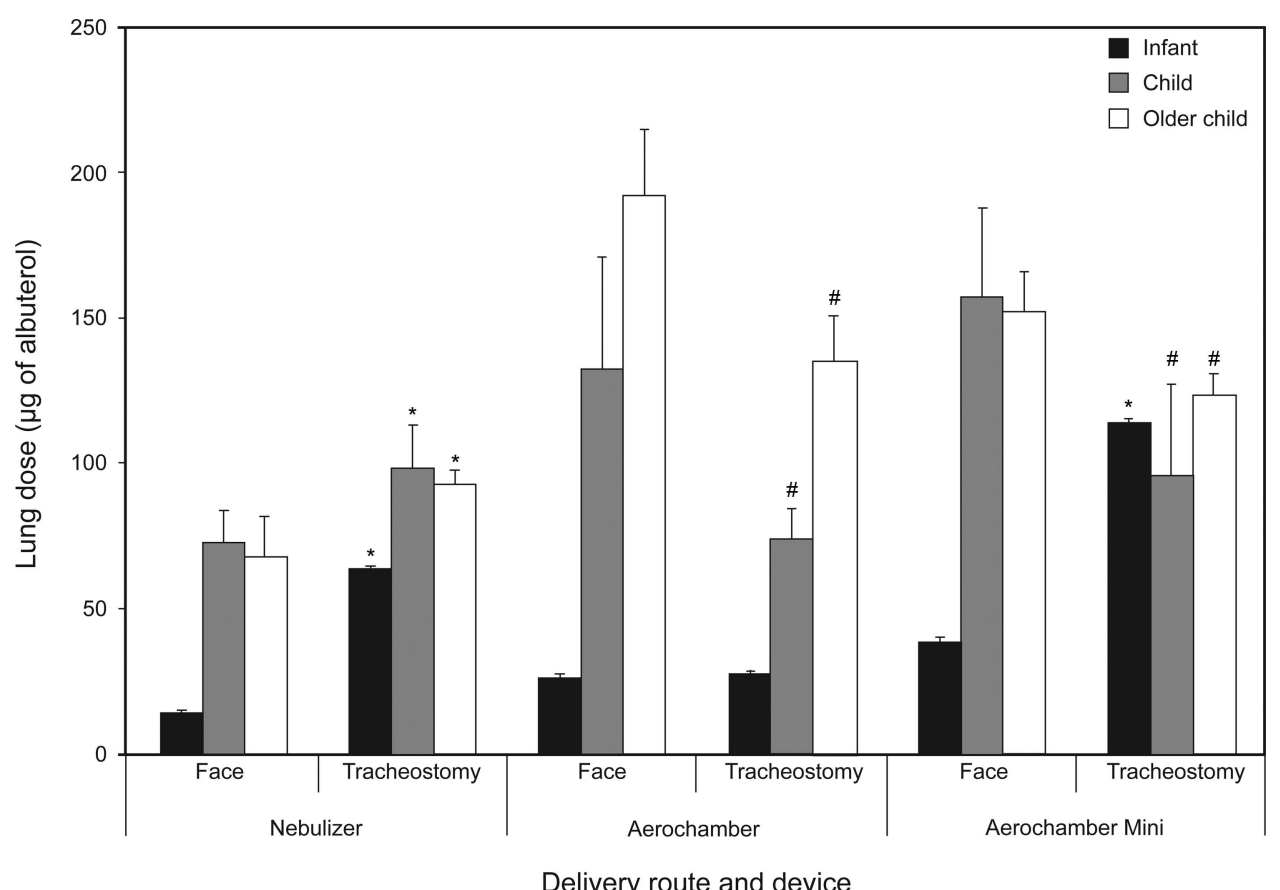

Fig. 5. Lung dose achieved with different devices and breathing patterns during facial and tracheostomy albuterol delivery. Error bars denote SD. *Dose delivered through tracheostomy is significantly higher than dose delivered through face mask $(P<.05)$. \# Dose delivered through tracheostomy is significantly lower than dose delivered through face mask $(P<.05)$.

\section{Use of the Assisted Technique}

During nebulization through the face, the use of the assisted technique resulted in a decrease in the lung dose in the child breathing pattern (tidal volume $155 \mathrm{~mL}$ ) $(P=.03)$ and in no changes for the infant (tidal volume $50 \mathrm{~mL}$ ) and older child (tidal volume $300 \mathrm{~mL}$ ) breathing patterns $(P=.19$ and $P=.53$ respectively) (Table 1). During nebulization through the tracheostomy, the use of the assisted technique resulted in an increase in the older child breathing pattern $(P=.02)$ and in no changes in the infant and child breathing patterns $(P=.33$ and $P=.22$, respectively).

During albuterol delivery with pMDI and AeroChamber Mini VHC through the face, the use of the assisted technique resulted in a decrease of the lung dose for the child and older child breathing patterns $(P=.02$ and $P=.02$, respectively) and in no change for the infant breathing pattern $(P=.77)$. During albuterol delivery with the pMDI and AeroChamber Mini VHC through the tracheostomy, the use of the assisted technique resulted in a decrease in the lung dose for the infant breathing pattern $(P=.01)$ and in no change for the child and older child breathing patterns $(P=.85$ and $P=.10$, respectively).

\section{Amount of Drug Deposited in the Tracheostomy Tube}

During nebulization, the amount of albuterol retained in the tracheostomy tube was $4.4,3.9$, and $1.2 \%$ of the nominal dose for the infant (tidal volume $50 \mathrm{~mL}$ ), child (tidal volume $155 \mathrm{~mL}$ ), and older child (tidal volume $300 \mathrm{~mL}$ ) 
breathing patterns. The use of the assisted technique led to a reduction in the amount of drug retained in the tube of 80, 60, and $40 \%$ for the infant, child, and older child breathing pattern, respectively.

When the pMDI and AeroChamber Mini VHC were used, the amount of albuterol retained in the tracheostomy tube was $6,13.9$, and $19.2 \%$ of the nominal dose for the infant, child, and older child breathing pattern, respectively. The use of the assisted technique led to a 9-, 5-, and 3 -fold increase in the amount of albuterol retained in the tracheostomy tube for the infant, child, and older child breathing pattern, respectively.

\section{Particle Size}

Administering an albuterol pMDI with an AeroChamber Mini VHC through the tracheostomy decreased its MMAD from 2.14 to $1.65 \mu \mathrm{m}(P=.002)$. Administration through the face also led to a decrease in $\operatorname{MMAD}(1.90 \mu \mathrm{m})$, but the difference was not statistically significant $(P=.054)$. Administering albuterol pMDI with an AeroChamber VHC decreased its MMAD $(2.15 \mu \mathrm{m})$ when administered through the face $(1.89 \mu \mathrm{m}, P=.045)$ and through the tracheostomy (1.74 $\mu \mathrm{m}, P=.007)$. No differences were found between both devices at all tested conditions $(P=.86, P=.91$, and $P=.31$ for pMDI with VHC alone, pMDI with VHC administered though the face, and pMDI with VHC administered through the tracheostomy, respectively).

No differences in geometric SD were found between all tested conditions with the AeroChamber Mini VHC (1.44, 1.44 , and 1.37 with $P=.99, P=.23$, and $P=.21$ for pMDI with VHC alone, pMDI with VHC administered though the face, and pMDI with VHC administered through the tracheostomy, respectively). No differences in geometric SD were found between all tested conditions with the AeroChamber VHC $(1.46,1.86$, and 1.25 with $P=.47, P=.80$, and $P=.30$ for pMDI with VHC alone, pMDI with VHC administered though the face, and pMDI with VHC administered through the tracheostomy, respectively). No differences were found between both devices at all tested conditions $(P=.82, P=.44$, and $P=.10$ for pMDI with VHC alone, pMDI with VHC administered though the face, and pMDI with VHC administered through the tracheostomy, respectively). The percentage of drug mass associated with particles $<5 \mu \mathrm{m}$ was $>99 \%$ for both devices at all tested conditions.

\section{Equivalence Between Nebulizer and pMDI Therapy}

During facial delivery, a unit dose of $2.5 \mathrm{mg} / 3 \mathrm{~mL}$ was equivalent to 4-5 actuations of a pMDI and either VHC (Table 3). However, during delivery through a tracheostomy, the AeroChamber Mini (5-8 actuations) was more efficient than the AeroTrach (7-25 actuations).

\section{Discussion}

We found that the effect of changing the delivery route from face to tracheostomy was variable and depended on the delivery device and the breathing pattern. We also found no advantage in using the assisted technique to enhance aerosol delivery. The particle size of the pMDI decreased after traveling through a pediatric tracheostomy tube. A reduction in the ID of a tracheostomy tube impaired aerosol delivery.

The finding that changing the delivery route of nebulized albuterol from facial to through the tracheostomy (tidal volumes ranging from 50 to $300 \mathrm{~mL}$ ) increased the lung dose is not in agreement with the findings in an adult study by Pitance et al. ${ }^{3}$ Our data showed a large increase in the lung dose with the small tidal volume (4.4-fold), whereas with other tidal volumes, the increase was small (1.3-1.4-fold). They evaluated lung deposition of nebulized amikacin delivered through a mouthpiece and a tracheostomy route by measuring urinary amikacin. The study comprised 9 subjects (age 50-64 y) with tracheostomies with an ID of $8.5 \mathrm{~mm}$ ( 8 of 9 subjects). Breathing frequency and tidal volume were $20 / \mathrm{min}$ and $540 \mathrm{~mL}$ and $17 / \mathrm{min}$ and $600 \mathrm{~mL}$ during nebulization through the tracheostomy and through a mouthpiece, respectively. Several reasons could explain the different results, including tidal volume, ID of the tracheostomy tube, and setting (in vitro vs in vivo). We found no difference in the lung dose between the breathing patterns with 155 and $300 \mathrm{~mL}$, suggesting that there might be a threshold value of tidal volume that would determine a higher lung dose. In addition, during inhalation of nebulized drugs, the lower the inspiratory flow, the higher the proportion of undiluted nebulized drug that the patient/model will receive. Also, a larger ID would minimize the detrimental effect of artificial airways on aerosol delivery. Previous in vitro studies have demonstrated the inverse relationship between the tracheostomy tube ID and lung dose. ${ }^{7,8} \mathrm{We}$ also found that the presence of a tracheostomy did not impair nebulized drug delivery through the facial route when a breathing pattern with a tidal volume of $150 \mathrm{~mL}$ was used. The results with the pMDI varied according to the type of VHC used. The large volume VHC had a similar dose for the lower tidal volume but had a decrease in the lung dose with the larger tidal volumes. The small volume VHC showed a several-fold increase in the lung dose with the low tidal volume. We speculate that these differences could be related to the relationship between the tidal volume and the volume of the VHC. The efficiency could be diminished during larger tidal volumes because an increase in flow creates more impaction in the artificial airways, as found in this study. Drug delivery with the pMDI and VHC was impaired with the AeroChamber but not with the AeroChamber Mini. We speculate that the difference 
in behavior could be due to the different volumes of the VHCs.

Our findings regarding nebulization through facial and tracheostomy routes and breathing patterns are in agreement with previous reports. ${ }^{7,14}$ The breathing pattern with the lowest tidal volume $(50 \mathrm{~mL})$ had a lung dose that was lower than with the other breathing patterns (155 and $300 \mathrm{~mL}$ ), which were similar. This could be explained by the fact that both breathing patterns had an inspiratory flow (tidal volume/inspiratory time $\times 60$ ) that exceeded the nebulizer flow (11.6 and $22.5 \mathrm{~L} / \mathrm{min}$, respectively).

During albuterol delivery through the face with a pMDI, the lung dose was lower for the 50 -mL tidal volume breathing pattern than for others. However, once the tidal volume exceeded the volume of the chamber, no further improvement in the lung dose was noted. During tracheostomy delivery with the pMDI, findings were similar. These results for the small volume VHC are in agreement with previously published data using a different experimental setup. ${ }^{6}$ A comparison between both studies shows a 2-fold higher lung dose for the previous model, which would be clinically equivalent to inhalation through the tracheostomy with the mouth closed. This underscores the importance of interpreting results of in vitro studies in light of their experimental setup.

The findings that during tracheostomy delivery, a change in ID from 4.5 to $3.5 \mathrm{~mm}$ decreased the lung dose in the breathing pattern with a small tidal volume is consistent with previous reports. ${ }^{6}$ In a previous study using another 2-compartment model, the lung dose decreased by a similar proportion in both VHCs when using a pMDI with the VHC. ${ }^{6}$ The large volume VHC had a decrease that was 3 -fold higher than the one seen with the small volume VHC. These findings need to be taken into account by the practitioner when deciding what device to recommend. The data for nebulizer therapy were similar to pMDI and also consistent with previous reports in tracheostomy and endotracheal tube models. ${ }^{7,8,15}$

The use of the assisted technique was evaluated both through the facial and the tracheostomy route. Aerosol delivery through the face with a nebulizer and a pMDI with a VHC was either unchanged or impaired by the use of this technique. We speculate that this was due to an increase in turbulence and subsequent aerosol impaction against the devices and face model. There are no previously published data to compare. Our findings suggest that the use of this technique during facial delivery is futile. The use of the assisted technique with a nebulizer through the tracheostomy led to a modest increase in the lung dose that only achieved statistical significance for the 300-mL tidal volume breathing pattern (17\%). These findings are in partial agreement with previous studies. ${ }^{6,7,9}$ Alhamad $^{9}$ compared tracheostomy delivery with the assisted and unassisted technique of a pMDI with spacer, a jet nebulizer, and a vibrating mesh nebulizer and found no differences in the lung dose. The author used a single-compartment model and respiratory settings similar to the $150 \mathrm{~mL}$ that we used. In a previous study using a 2-compartment model, the use of the assisted technique for nebulizer resulted in an increase in the lung dose. Another study using an adult model with a tidal volume of $450 \mathrm{~mL}$ found a 3.5 -fold increase in the lung dose when the assisted technique was used with a nebulizer. ${ }^{5}$ These results are influenced by the experimental setup, which included the use of a larger tracheostomy tube (ID $=8 \mathrm{~mm}$ ), a passive test lung, and a single-compartment model. The use of the assisted technique with the pMDI resulted in a decrease in the lung dose for the lower tidal volume and was not changed for the $155-$ and $300-\mathrm{mL}$ breathing patterns. These data are in agreement with those of Alhamad ${ }^{9}$ for the $155 \mathrm{~mL}$ tidal volume and with our study ${ }^{6}$ for the breathing pattern with the low tidal volume. In our previous study, ${ }^{6}$ the use of the assisted technique with the pMDI impaired drug delivery for all breathing patterns. The difference in setup between both studies could explain in part the different outcome. Although both studies used a 2-compartment model, the previous study used a one-way valve at the upper airway level that opened on expiration. The other difference was that the operators of the assisted technique were different.

The amount of drug retained by the tracheostomy tube $(1.2-4.4 \%)$ was consistent with previous reports that found $0.8 \%$ in a pediatric model and $2-16 \%$ in an adult model. ${ }^{7,8}$ It was noticed that the amount decreased with increasing tidal volume. Conversely, the amount deposited with the pMDI increased with increasing tidal volumes. We speculate that this different behavior could be explained in part by the fact that the higher inhalation flows achieved with the breathing pattern with larger tidal volumes led to increased impaction in the pMDI due to the high speed of the aerosol. The amount of drug deposited in the tube when pMDIs were used was higher than previously reported in a 2-compartment pediatric model $(7.8 \%){ }^{6}$ The difference could be explained in part by the fact that a slower pMDI (Proair hydrofluoroalkane) was used in the study that reported lower deposition. ${ }^{16}$

The findings of the change in particle size of a pMDI aerosol that traveled through tracheostomies is consistent with previous reports on nebulized aerosol. ${ }^{7}$ However, the effect was smaller due to the fact that the MMAD for the pMDI $(2.14-2.15 \mu \mathrm{m})$ was smaller than that of the nebulizer $(4.56 \mu \mathrm{m})$. Similarly, the characterization of the aerosol using an anatomically correct airway led to a decrease in particle size as reported previously for nebulizers. ${ }^{11}$ We speculate that the use of aerosols with a MMAD closer to $1 \mu \mathrm{m}$ will result in an increase in delivery efficiency.

This study allowed us to compare the doses and analyze the equivalent dose ranges for each device and delivery 
route. We found that in agreement with previously published data, a unit dose of albuterol of $2.5 \mathrm{mg} / 3 \mathrm{~mL}$ is equivalent to $4-5$ puffs during facial delivery. ${ }^{17} \mathrm{We}$ found that during pMDI delivery through a tracheostomy, a small volume VHC was more efficient than a large volume VHC. These results revealed a difference between nebulizer and pMDI with VHCs that was larger than previously described. ${ }^{6,7,9}$ Previous studies using either single-compartment or 2-compartment models reported a dose equivalency of 3 actuations with a $2.5 \mathrm{mg} / 3 \mathrm{~mL}$ albuterol unit dose. This marked difference with previous reports resulted from the improved performance of nebulizers and the decreased performance of the VHCs with this model.

The major limitation of this study is that it was not an in vivo study, therefore not taking into account the biological processes the drug would undergo. Although the methods used were well established in aerosol research, they are known to overestimate actual drug delivery. The findings of this study are limited to the experimental conditions. There should be no extrapolation of data to either smaller size tracheostomy tubes or breathing patterns with lower tidal volumes.

\section{Conclusions}

The series of experiments reported in this study should help practitioners to optimize drug delivery to spontaneously breathing tracheostomized pediatric patients and should also help them to determine whether dose changes are necessary when changing from a facial to tracheostomy route or vice versa. Whereas a decrease in nebulized dose might be necessary, conversely, an increase in pMDI might be required when changing from the facial to the tracheostomy route. In addition, guidance is provided regarding the equivalence of albuterol delivered via nebulization and pMDI/VHC. Practitioners should not use assisted delivery with the intent of enhancing drug delivery. Practitioners should be mindful that during downsizing of the tracheostomy, aerosol delivery will be impaired. The particle size of a pMDI decreased after traveling through pediatric tracheostomy tubes, and we speculate that aerosols with a smaller particle size could be delivered more efficiently through them. Animal and human studies are necessary to establish in vivo/in vitro correlations.

\section{REFERENCES}

1. Sherman JM, Davis S, Albamonte-Petrick S, Chatburn RL, Fitton C, Green C, et al. Care of the child with a chronic tracheostomy. Am J Respir Crit Care Med 2000;161(1):297-308.

2. Amirav I, Newhouse MT. Aerosol therapy in tracheotomized children: time for guidelines! Respir Care 2012;57(8):1350.

3. Pitance L, Reychler G, Vecellio L, Leal T, Reychler H, and Liistro G. Influence of tracheostomy on lung deposition in spontaneously breathing patient. Pitance $\mathrm{L}, \mathrm{PhD}$ thesis, Catholic University of Louvain, 2013;117-132.

4. Piccuito CM, Hess DR. Albuterol delivery via tracheostomy tube. Respir Care 2005;50(8):1071-1076.

5. Ari A, Harwood RJ, Sheard MM, and Fink JB. An in-vitro evaluation of aerosol delivery through tracheostomy and endotracheal tubes using different interfaces. Respir Care 2012;57(7):1066-1070.

6. Berlinski A, and Chavez A. Albuterol delivery via metered dose inhaler in a spontaneously breathing pediatric tracheostomy model. Pediatr Pulmonol 2013;48(10):1026-1034.

7. Berlinski A. Nebulized allbuterol delivery in a model of spontaneously breathing children with tracheostomy. Respir Care 2013;58(12): 2076-2086.

8. Pitance L, Vecellio L, Delval G, Reychler G, Reychler H, Liistro G. Aerosol delivery through tracheostomy tubes: an in vitro study. J Aerosol Med Pulm Drug Deliv 2013;26(2):76-83.

9. Alhamad B. The effect of aerosol devices and administration techniques on drug delivery in a simulated spontaneously breathing pediatric model with a tracheostomy. MS thesis, Georgia State University, Atlanta, GA, 2013.

10. Willis LD, Berlinski A. Survey of aerosol delivery techniques to spontaneously breathing tracheostomized children. Respir Care 2012; 57(8):1234-1241.

11. Berlinski A, Xi J. Characterization of nebulized aerosols using a next generation impactor and an anatomically correct pediatric airway model. Respir Drug Deliv 2014;3:637-640.

12. Chavez A, McCracken A, Berlinski A. Effect of face mask static dead volume, respiratory rate and tidal volume on inhaled albuterol delivery. Pediatr Pulmonol 2010;45(3):224-229.

13. Marple VA, Olson BA, Santhanakrishnan K, Mitchell JP, Murray SC, Hudson-Curtis BL. Next generation pharmaceutical impactor (a new impactor for pharmaceutical inhaler testing). Part II: Archival calibration. J Aerosol Med 2003;16(3):301-324.

14. Berlinski A. Effect of mask dead space and occlusion of mask holes on delivery of nebulized albuterol. Respir Care 2014;59(8):12281232 .

15. Ahrens RC, Ries RA, Popendorf W, Wiese JA. The delivery of theraputic aerosols through endotracheal tubes. Pediatr Pulmonol 1986;2(1):19-26.

16. Hautmann J, Godoy SE, Marshik P, Chand R, McConville J, Krishna $\mathrm{S}$, et al. Effect of time between actuation on the dose variability for three metered dose inhalers. Respir Drug Deliv Eur 2013;2:429-434.

17. EPR-3. Expert Panel Report 3: guidelines for the diagnosis and management of asthma (EPR-3 2007). NIH Publication No. 085846. Bethesda, MD: National Institutes of Health; NHLBI; National Asthma Education and Prevention Program: 2008.

This article is approved for Continuing Respiratory Care Education credit. For information and to obtain your CRCE

(free to AARC members) visit www.rcjournal.com

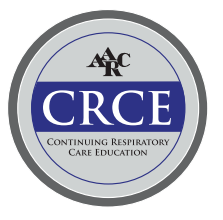

